\title{
Post Traumatic Hemobilia: Three Case Reports
}

\author{
Hicham Jalal'1, Hana Elmansouri'1, Sofia El Fakir'1, Leila Berghalout' ${ }^{1}$, I. Zouita ${ }^{1}$, \\ Najat Cherif Idrissi El Ganouni'1, Youssef Narjiss ${ }^{2}$, Khalid Rabbani \\ ${ }^{1}$ Department of Radiology, University Hospital Mohammed VI, Marrakesh, Morocco \\ ${ }^{2}$ Department of Visceral Surgery, Faculty of Medicine and Pharmacy of Marrakesh, Cadi Ayyad University, Marrakesh, Morocco \\ Email: hana.elmansouri@gmail.com
}

How to cite this paper: Jalal, H., Elmansouri, H., El Fakir, S., Berghalout, L., Zouita, I., El Ganouni, N.C.I., Narjiss, Y. and Rabbani, K. (2019) Post Traumatic Hemobilia: Three Case Reports. Open Access Library Journal, 6: e5102.

https://doi.org/10.4236/oalib.1105102

Received: December 10, 2018

Accepted: January 8, 2019

Published: January 11, 2019

Copyright $\odot 2019$ by author(s) and Open Access Library Inc.

This work is licensed under the Creative

Commons Attribution International

License (CC BY 4.0).

http://creativecommons.org/licenses/by/4.0/

\begin{abstract}
The authors report three cases of post-traumatic hemobilia. Hemobilia complicates about $0.5 \%$ to $2 \%$ of liver injuries. The diagnosis can be difficult because of its possible delay of appearance. The ultrasound and CT scan performed urgently are of great help in both positive diagnosis and monitoring of this pathology. Once the diagnosis is made-immediatly or a while after the trauma-a diagnostic and therapeutic arteriography must be carried out quickly.
\end{abstract}

\section{Subject Areas}

Radiology \& Medical Imaging

\section{Keywords}

Hemobilia, Abdominal Trauma, CT, Arteriography

\section{Introduction}

Hemobilia is a bleeding in the bile ducts due to a communication between the biliary tree and an intra or extra-hepatic vessel [1]. It is a rare condition of various etiologies. Post-traumatic hemobilia is a rare complication of abdominal trauma [2] and its clinical expression may be delayed. Imaging, mainly ultrasound and CT, allows the positive diagnosis. Arteriography has double use since it ensures the etiological diagnosis and the therapeutic management when it is possible [2].

\section{Observations}

Case $\mathrm{N}^{\circ} 1$ :

36-year-old patient, victim of an abdominal trauma with epigastric impact. 
On admission, the patient was in good hemodynamic state, clinical examination found an epigastric and right hypochondrium guarding. Ultrasound and initial CT showed poorly limited hyperechoic lesions of segments IV, V, VII and VIII related to foci of bruising (Figure 1). The patient received symptomatic treatment with monitoring. The evolution was marked by the appearance-eight days later-of hematemesis of average abundance and hematochezia with a state of shock.Endoscopy did not find any oeso-gastroduodenal lesion. Control CT showed in addition to hepatic contusion, a dilatation with hemorrhage of the intrahepatic bile ducts and gall bladder. After contrast agent injection, the gall bladder and the bile ducts were opacified (Figure 2 and Figure 3). Mesenteric angiography did not show extravasation of the contrast agent. The evolution was good under clinical and biological monitoring.

\section{$>$ Case $\mathrm{N}^{\circ}$ 2:}

53-year-old patient, polytrauma with abdominal impact. On admission, the patient was in a good hemodynamic state, presenting an epigastric and right hypochondrium guarding. Initial abdominal ultrasound and CT showed fracture of the IV segment of the liver with contusions of segments VI and VII (Figure 4). The evolution noted the appearance-six days later-of mucocutaneous

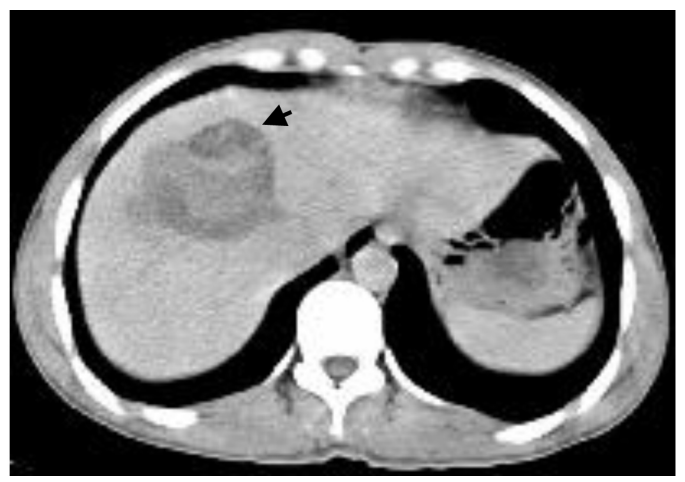

Figure 1. Abdominal CT. Axial image shows aheterogeneoushypo dense lesion in segment IV and VII relevant to hepatic contusion.

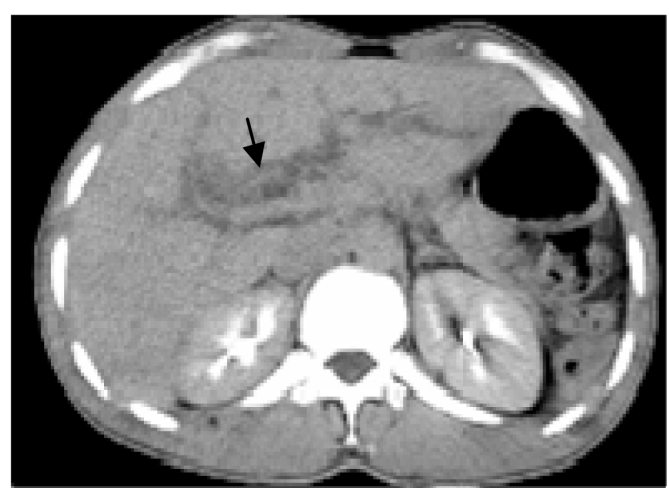

Figure 2. Abdominal CT. Axial image shows high-attenuation clot within the dilated intra hepatic bile ducts (arrow). 


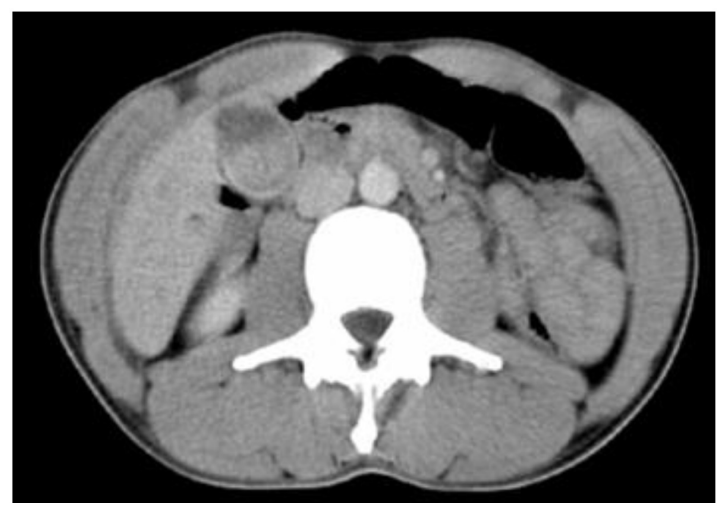

Figure 3. Contrast enhanced CT shows enhancement of gallbladder content and wall.

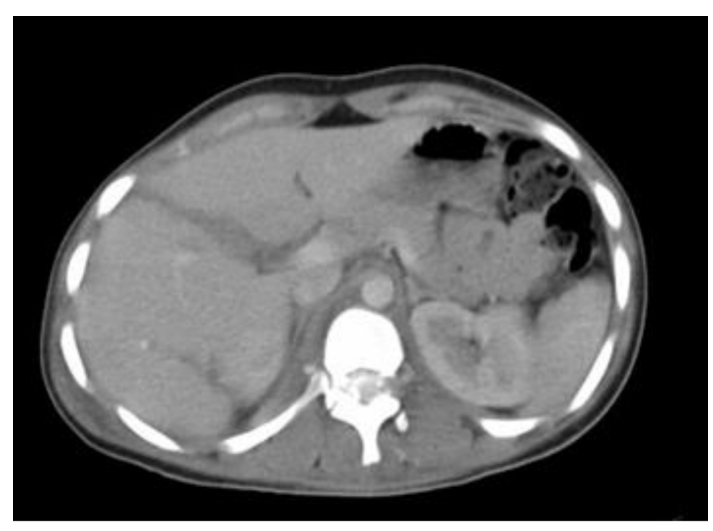

Figure 4. Contrast enhanced abdominal CT showing Hepatic Fracture in segment IV and VI.

jaundice with hematemesis of low abundance, melena and fever at $38^{\circ}$. Control CT showed peri-hepatic peritoneal effusion on segment IV of the liver. The gallbladder had an intra-mural hematoma (Figure 5). Treatment was based antibiotics and monitoring. One month after the trauma, the patient presented pain in the hypochondrium and right flank with vomiting. The clinical examination found a guarding of the right hemi-abdomen with fever at $38.8^{\circ} \mathrm{C}$. A third CT scan showed a large biliary collection of the liver extending from the para-colic gutter to the right iliac fossa. This collection was spontaneously hypodense, surrounded by a wall non-enhanced by the contrast agent suggesting a biloma. Hepatic contusions of segments IV and V had been decreased. Percutaneous drainage of the biloma allowed total recovery.

\section{$>$ Case $\mathrm{N}^{\circ} 3$ :}

24-year-old patient, admitted to the emergency department for a polytrauma caused by a collapsed roof. On admission, clinical examination found a conscious patient in good hemodynamic state with paraplegia of both lower limbs and an umbilicus sensory level. CT scan of the spine showed a fracture-dislocation of D12 and L1 with intra-canal fragment. The emergency ultrasound showed a gallbladder of normal size, regularly thickened wall, has an 


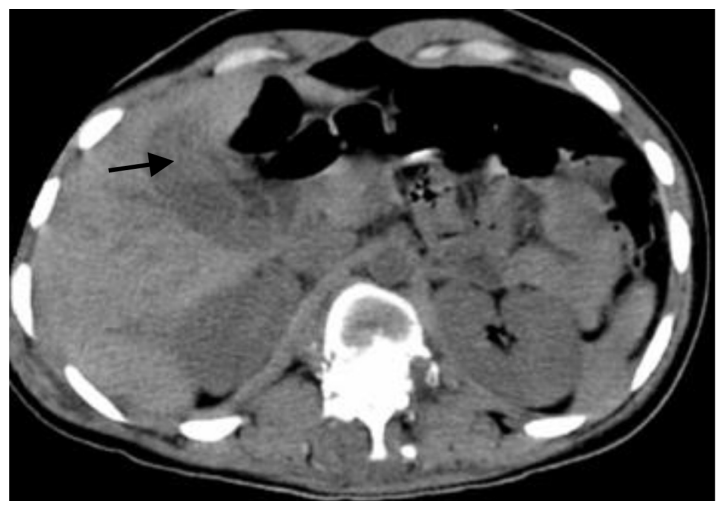

Figure 5. Abdominal CT with and without contrast. Axial images showing high-attenuation material within the gallbladder (arrow): hemobilia.

echoic image of cone without cast shadows suggesting a hematoma. Complementary CT confirmed the diagnosis of hemobilia by showing a spontaneously hyperdense image in the gallbladder lumen not enhanced by contrast agent, associated with hepatic fracture and hemorrhagic contusion of segments IV, V and VIII. The initial biological assessment was normal. The treatment based on clinical and biological monitoring was with good evolution.

\section{Discussion}

Hemobilia is a pathological communication between the bile duct and an intra or extrahepatic vessel, most often arterial than portal [3]. It complicates $0.5 \%$ to $2 \%$ of closed hepatic injuries, most often by lesion of a Glissonean pedicle leading to the constitution of an arterial pseudoaneurysm, then its rupture in the correspondent bile duct [3]. The interval between hemobilia and hepatic contusion is on average three to four weeks. Its frequency is increasing because of traffic accidents and the use of invasive investigations in the diagnosis and treatment of hepatobiliary affections. The latter is responsible for $10 \%$ of hemobilia [4] [5] and is most often secondary to rupture of aneurysm of the hepatic artery of atheromatous origin [4] [6].

The clinical signs of hemobilia are related to upper gastrointestinal bleeding and biliary obstruction by blood clots and are therefore showed by the Sandblom triad, combining: Biliary colic (70\%), jaundice (60\%), externalized digestive haemorrhage made of hematemesis $(60 \%)$ and melena in $90 \%$ of cases. These three signs can be combined in $30 \%$ of cases [2]. The long interval between trauma and symptoms can reach several months explaining the delayed diagnosis noticed in our first two patients. In some cases, the diagnosis is immediately suggested in front of a direct vesicular contusion like the case of our third patient [7]. Biology may show signs of early hepatocellular disease [2] [4]. Upper gastrointestinal endoscopy (UGIE) is the test of choice for the exploration of upper gastrointestinal bleeding [2] [4] [5]. If it visualizes blood or a clot extruding from the Ampulla of Vater, it contributes to the diagnosis of hemobilia. 
Nevertheless, this test confirms the diagnosis in only $12 \%$ of cases because blood clots can block the bile duct and make the bleeding discontinuous [4]. A sphincterotomy and clot extraction allows relieving the symptoms [4].

In our first two patients the UGIE could not objectify the origin of the digestive bleeding. Ultrasound, often performed urgently, is of great benefit in the positive diagnosis of this pathology. Indeed, as our third observation illustrates, it allows the visualization within the lumen of the gallbladder of an irregular echogenic conestructure without cast shadows suggesting an intra luminal hematoma [1] [5] [7] [8].

However, this aspect may be confusing with other diagnoses such as biliary mud, polyp or tumor, purulent debris or parasitic remains [2]; it also allows showing a dilation of Intrahepatic bile ducts, hyperechoic clots in bile duct or intrahepatic hematoma. CT is not a sensitive test for the diagnosis of hemobilia, nevertheless it allows to highlight spontaneously hyperdense hemorrhage within the gallbladder (hemocholecyst) and bile ducts that can be dilated. But again, such an image can also mark a problem of differential diagnosis with micro-stones or calcium sludge.

Highlighting opacification of the bile ducts by the CT is pathognomonic to confirm arterio-biliary fistula. Arteriography is the best examination, both for its diagnostic (and topographical) and therapeutic input. It has a sensitivity of $90 \%$ [3]. The classic image of the passage of the contrast agent in bile ducts is pathognomonic but rare and often it is a pseudoaneurysm [2] [3]. Because of frequent anatomical variants, a celiac and mesenteric exploration is recommended.

The causes of false-negatives are; absence of active bleeding, abnormalities of the origin of the hepatic arteries and history of liver surgery [3]. Arteriography may be normal as was the case of our first patient. Complications of hemobilia depend on; severity of bleeding as well as level of obstruction of bile ducts by blood clots. These complications are: haemorrhagic shock, cholecystitis by haemocholecyst, cholangitis, or rarely pancreatitis [3].

\section{Conflicts of Interest}

The authors declare no conflicts of interest regarding the publication of this paper.

\section{References}

[1] Karsenti, D., Tchuenbou, J. and Djarech, H. (2002) Pancréatite aiguë liée à une hémobilie compliquant un carcinome hépatocellulaire. Gastroentérologie Clinique et Biologique, 26, 1051-1054.

[2] Echarrab, M., Mdeghri, J. and El Ounani, M. (2000) Hémobilie : Quel Traitement. Médecine du Maghreb, 80, 32-34.

[3] Rousseau, A., Regimbeau, J.M., Vibert, E., Vullierme, M.P., Sauvanet, A. and Belghiti, J. (2004) Hémobilie après traumatisme hépatique fermé: Une complication parfois tardive [Haemobilia after Blunt Hepatic Trauma: A Sometimes Delayed Complication]. Annales de Chirurgie, 129, 41-45. 
https://doi.org/10.1016/j.anchir.2003.11.003

[4] Iannelli, A., Karimdjee, B. and Fabiani, P. (2003) Hémobilie par pseudoanévrysme de la branche droite de l'artère hépatique après cholécystectomie coelioscopique. Gastroentérologie Clinique et Biologique, 27, 341-343.

[5] Barthet, M. and Heyries, L. (2002) Complications à court terme de la CholangioPancréatographie rétrograde endoscopique diagnostique et thérapeutique. Gastroentérologie Clinique et Biologique, 26 , 980-987.

[6] Lesur, G. (2005) Hémorragies digestives hautes de causes rares. Gastroentérologie Clinique et Biologique, 29, 682-94. https://doi.org/10.1016/S0399-8320(05)82157-8

[7] Gomez, M.A., Couthon, A., Gilbert, B., Watteau, N., Roger, R. and Scotto B. (2002) Contusion vésiculaire isolée Post-Traumatique. Journal de Radiologie, 83, 490-491.

[8] Petit, Ph., Portier, F. and Devred, Ph. (1999) Échographie et traumatisme abdominal chez l'enfant. Journal d Echographie et de Médecine du Sport, 20, 169. 